\title{
APOYO ACADÉMICO EN LA EDUCACIÓN SUPERIOR: DESCRIPCIÓN DE LA EXPERIENCIA Y PERFIL DE LOS ALUMNOS QUE PARTICIPAN EN EL CARA UC
}

\section{Introducción}

Los objetivos de este artículo son, por una parte, dar a conocer la experiencia del Centro de Apoyo al Rendimiento Académico para los alumnos de la Pontificia Universidad Católica de Chile (CARA UC) y, por otra, describir las características sociodemográficas, antecedentes académicos y variables afectivo-motivacionales asociadas al rendimiento de los alumnos que participan de forma espontánea en el Centro. Además, se describen las principales motivaciones que llevan a los alumnos a consultar y los servicios de apoyo que solicitan. La evidencia indica que, a pesar de que los jóvenes que ingresan a la Pontificia Universidad Católica de Chile (PUC) tienen, en general, antecedentes de excelencia académica y buen rendimiento (comparado con otras universidades) (Valdivieso, Antivilo y Barrios, 2006), la cantidad de alumnos que acude al Centro en busca de apoyo educativo es muy alta y se aprecian demandas variadas. En este contexto, la principal pregunta tiene relación con las características particulares de estos alumnos. Existen antecedentes de que otras instituciones de educación superior del país también se han visto enfrentadas a la necesidad de ofrecer a sus estudiantes servicios de apoyo similares a los del CARA UC. En este sentido, el estudio también pretende dar a conocer la experiencia de intervención realizada por el Centro como una alternativa para abordar las necesidades académicas de los alumnos y que éstos logren mantenerse en la universidad y culminar con éxito sus estudios.

A continuación se describen antecedentes generales respecto de la creación de espacios de apoyo y orientación académica a los 
310 APOYO ACADÉMICO EN LA EDUCACIÓN SUPERIOR - María Inés Susperreguy, Alejandra Flores, Sonia Micin, M. Soledad Zuzulich

alumnos universitarios, y, en particular, se presenta la experiencia y modo de trabajo del CARA UC. Específicamente, se muestra el proceso de creación del Centro, los objetivos que persigue, los servicios que ofrece y algunos indicadores generales respecto de su funcionamiento. Posteriormente, se detallan las principales características de la población que ha consultado y participado de los distintos servicios ofrecidos. Por último, se plantean algunos temas de discusión y las posibles proyecciones del Centro en atención a los resultados del estudio.

\section{Antecedentes de la experiencia del CARA UC}

La transición de la enseñanza secundaria a la educación superior enfrenta al estudiante a múltiples desafíos, tanto en el ámbito académico como personal. Se ve obligado a poner en marcha nuevas habilidades cognitivas, estrategias de aprendizaje y conductas académicas que le permitan sortear sin mayores dificultades el ingreso y permanencia en la universidad, lo cual supone mayores exigencias que van en aumento (Bilbao, 2004).

Pese a que algunos son exitosos en responder a los desafíos que depara la universidad, un grupo considerable de alumnos universitarios chilenos no ha adquirido ni desarrollado a cabalidad en la etapa escolar las habilidades y conductas académicas indispensables para enfrentar con éxito las nuevas demandas cognitivas y actitudinales (Polo, Hernández y Pozo, 1996). Los estudios realizados con estudiantes universitarios muestran una escasa frecuencia en el uso de técnicas complejas de estudio y dificultades para autorregular su conducta y esfuerzo de manera sistemática, características indispensables para desempeñarse exitosamente en el ámbito universitario y obtener un buen rendimiento (García y Ramírez, 2005). Algunos de ellos pueden desarrollar síntomas físicos y psicológicos en el transcurso de su carrera (Andrews y Wilding, 2004; Díaz, Prado y López, 2003; Villarroel, 2001; Malenic y Ortega-Soto, 1995) y, si estas perturbaciones se mantienen, se pueden traducir en calificaciones deficientes, reprobación de asignaturas y, en algunos casos, deserción del sistema universitario (Polo et al., 1996). 
El bajo rendimiento en algunas carreras o cursos específicos de las mallas curriculares y la deserción estudiantil afectan de manera generalizada a las universidades chilenas, constituyendo un problema nacional, ya que implica una pérdida de capital de recursos humanos, tanto para la familia del alumno como para la universidad y el país (Alvarado, Sánchez y Uribe, 2000; Brunner y Elacqua, 2003; Consejo Superior de Educación, 2007; Sanabria, 2002). También, pone en evidencia la necesidad de profundizar en aquellos aspectos que inciden en el proceso enseñanza-aprendizaje al interior de la universidad, con el fin de permitir que todos los estudiantes obtengan un aprendizaje significativo y mejor rendimiento. Este escenario explica y justifica la generación de espacios de apoyo y orientación a los alumnos para que se adapten adecuadamente a los desafíos y exigencias académicas (Manelic y Ortega-Soto, 1995), e implementar programas en los cuales se les enseñe explícitamente nuevos hábitos, habilidades y estrategias de aprendizaje, así como técnicas de trabajo intelectual y conductas académicas que les permitan reflexionar acerca de su propio aprendizaje. La evidencia muestra efectos positivos en los alumnos que participan de estos cursos o programas de enseñanza y entrenamiento de habilidades. Ellos se traducen, por ejemplo, en la obtención de mejores puntajes en pruebas de inteligencia (Estévez, 2002) y en el desarrollo de habilidades de procesamiento profundo de la información, lo que facilita el trabajo académico y, por ende, potencia el rendimiento (Bilbao, 2004).

\section{La opción de la Pontificia Universidad Católica de Chile: el Centro de Apoyo al Rendimiento Académico (CARA UC)}

La misión del CARA UC es ofrecer diversas alternativas de crecimiento y desarrollo de habilidades académicas, para que los jóvenes vivan con éxito y satisfacción la etapa universitaria. Esta iniciativa responde a las políticas impulsadas por la rectoría, en orden a construir una universidad centrada en sus estudiantes y comprometida con los valores de excelencia y bienestar de todos sus alumnos, que 
312 APOYO ACADÉMICO EN LA EDUCACIÓN SUPERIOR - María Inés Susperreguy, Alejandra Flores, Sonia Micin, M. Soledad Zuzulich

se traducen en "generar instancias para desarrollar competencias cognitivas que mejoren el rendimiento académico y fortalezcan el ámbito de valores en los estudiantes" (Rosso, 2004). Se busca, además, "expandir los servicios de apoyo estudiantil en el área de estrés y bajo rendimiento académico, como también en crecimiento personal y orientación" (Rosso, 2004).

El CARA UC se constituye en un mecanismo para promover la equidad en la permanencia de los estudiantes una vez que han ingresado a la universidad. Por esta razón, se trata de una unidad gratuita y abierta a todos los alumnos, cuyo foco está puesto en la prevención del fracaso académico y el desarrollo de herramientas y recursos personales. Los alumnos asisten de manera voluntaria, a excepción de aquellos que participan en servicios incorporados en la malla curricular de algunas carreras o de intervenciones solicitadas por las facultades para alumnos en riesgo de eliminación por motivos académicos.

Se pone especial énfasis en la difusión de los servicios entre los estudiantes de los primeros años, ya que esta etapa es crucial para establecer bases sólidas, identificar a tiempo debilidades de aprendizaje y desplegar estrategias remediales con mayor probabilidad de éxito. (Gómez, Osorio, Domínguez, et al., 2002). Asimismo, se fomenta la comunicación con las unidades académicas que presentan altas tasas de reprobación y bajo rendimiento, y que reportan mayores dificultades, frustraciones y necesidades específicas en sus alumnos.

Depende de la Unidad de Apoyo Psicológico (UAP) de la Dirección General Estudiantil-Salud. Es una iniciativa que busca promover y desarrollar el bienestar integral de los alumnos, enfatizando variables relativas a la dimensión académica, en tanto éstas constituyen un factor protector de la salud mental. Su objetivo general es promover una adecuada adaptación a la vida universitaria y el bienestar biopsicosocial de los estudiantes, a través del fomento de actitudes y estrategias orientadas a fortalecer el rendimiento, ofreciendo diversas alternativas de apoyo acordes con sus necesidades, intereses y estilos de aprendizaje. 
De lo anterior se desprenden los objetivos específicos del CARAUC:

a) Generar conciencia acerca de la problemática del rendimiento académico en la comunidad universitaria, promoviendo una actitud positiva hacia el estudio como medio para lograr el aprendizaje.

b) Desarrollar y promover factores protectores de la salud mental relacionados con el quehacer universitario, tales como rendimiento, motivación, responsabilidad y percepción de autoeficacia.

c) Prevenir y/o pesquisar factores de riesgo que amenacen el bienestar integral de los alumnos: ansiedad, fracaso académico, falta de planificación, entre otros.

d) Fortalecer el desarrollo de habilidades y recursos personales para aprender, ofreciendo diferentes herramientas, dentro de un espacio voluntario, cooperativo, extracurricular, guiado y libre de calificaciones.

e) Apoyar y reforzar la formación académica que ofrecen las unidades académicas a sus estudiantes, con espacios grupales e individuales de desarrollo extracurricular, en algunas áreas o campos específicos del conocimiento.

f) Enriquecer el currículo mínimo de las facultades que lo soliciten, con la formación de actitudes y estrategias para el aprendizaje, a través del curso "Hábitos y estrategias de estudio" inserto en las mallas curriculares.

g) Apoyar psicoeducativamente a los alumnos que requieran atención y orientación individual en el desarrollo de habilidades para el aprendizaje.

h) Apoyar psicoeducativamente a los estudiantes que requieran participar en procesos de exploración y orientación vocacional.

i) Formar tutores alumnos en habilidades docentes y de liderazgo, que se destaquen en algunas de las áreas del conocimiento, promoviendo en ellos una actitud de responsabilidad social y de compromiso con su disciplina.

j) Diseñar programas remediales para grupos de alumnos que han estado en riesgo de eliminación. 
314 APOYO ACADÉMICO EN LA EDUCACIÓN SUPERIOR - María Inés Susperreguy, Alejandra Flores, Sonia Micin, M. Soledad Zuzulich

k) Mantener una coordinación entre área clínica y el CARA UC, con el fin de diagnosticar y derivar oportunamente a los alumnos entre los servicios.

1) Generar y compartir conocimiento en torno al rendimiento académico universitario, características y necesidades de apoyo de los estudiantes, estrategias de intervención exitosas y las actividades del CARA UC en general.

El diseño del proyecto se inicia a fines de 2003, buscando estrategias preventivas y remediales para los problemas de rendimiento y salud, asociados a los motivos de consulta de los alumnos en la Unidad de Apoyo Psicológico de la universidad. Se decide visitar y conocer consolidados centros de apoyo al aprendizaje en prestigiosas universidades de Estados Unidos (University of California, Berkeley, Miami, Stanford, U.C.L.A). El Centro inicia sus actividades en plan piloto el segundo semestre de 2004 y, desde su inauguración formal, a principios de 2005, ha convocado a más de mil participantes. En su tercer año de funcionamiento, CARA UC es un organismo reconocido al interior de la universidad, cuenta con una alta demanda de alumnos, diversificación de servicios e información de gestión que permite proyectar investigaciones y espacios para compartir esta experiencia.

Los servicios que se ofrecen responden tanto al análisis de los resultados estadísticos de rendimiento por ramos y carreras dentro de la universidad, como a los intereses que los mismos estudiantes van planteando, lo que permite ajustar la oferta de talleres, tutorías, atenciones y cursos según las posibilidades horarias de ellos. La idea es que éstos puedan escoger alternativas que no interfieran con sus compromisos académicos regulares.

Estos servicios comprenden:

1) "Test de habilidades académicas y estilo de aprendizaje", en línea en la página web de la universidad, "Inventario de Procesos de Estudio de los alumnos de la UC” (IPEUC) (Rosas, Manzi y Moore, 1987) e "Inventario de Estilos de Aprendizaje" (Kolb, 1976), para 
que los estudiantes puedan realizar un autodiagnóstico de su proceso de estudio.

2) Sesión diagnóstica de rendimiento: reunión grupal de una hora, con un máximo de 12 personas. Un psicólogo educacional interpreta y profundiza los resultados obtenidos por los alumnos en el test IPEUC. Se les entrega un plan de desarrollo individual con el diagnóstico y sugerencias específicas sobre los servicios del Centro. Al finalizar, los alumnos pueden inscribirse libremente en el servicio de su interés.

3) Talleres de habilidades académicas: los participantes aprenden y practican estrategias para el desarrollo de habilidades académicas específicas, tales como "Concentración y Memoria", "Planificación del tiempo", "Presentaciones orales", "Técnicas de estudio", "Lectura y Redacción". Los talleres tienen una extensión de tres a cinco sesiones, de tres horas cada una, con horarios variables para que los alumnos puedan acceder según su disponibilidad.

4) Tutorías académicas: asesorías técnicas en asignaturas específicas, conocidas por su grado de dificultad y altas tasas de reprobación. Están a cargo de alumnos-tutores -con desempeño de excelencia en estas asignaturas y con interés en desarrollar habilidades docentes- para explicar y resolver dudas teóricas y prácticas.

5) Atención psicoeducativa (counseling): espacio de acompañamiento individual, en el cual un psicólogo educacional orienta al alumno en el desarrollo de sus habilidades académicas y/o guía procesos de exploración vocacional. Previamente, los alumnos son entrevistados por un psicólogo clínico para pesquisar problemas emocionales y derivar a la atención clínica si corresponde.

6) Curso semestral de formación general "Hábitos y estrategias de estudio": se dicta como curso mínimo obligatorio para los alumnos de primer año en las facultades que lo soliciten, insertándolo en su malla curricular.

7) Intervenciones remediales focalizadas: se diseñan e implementan por petición de una carrera o facultad para una población particular que requiera desarrollar habilidades académicas específicas (por ejemplo, alumnos que han enfrentado una causal de eliminación 
316 APOYO ACADÉMICO EN LA EDUCACIÓN SUPERIOR - María Inés Susperreguy, Alejandra Flores, Sonia Micin, M. Soledad Zuzulich

y permanecen en la universidad con la condición de mejorar su rendimiento).

8) Actividades de extensión: cursos y talleres que se dictan fuera de la PUC, actividades de investigación, difusión, seguimiento de los alumnos participantes, entre otras.

Diagrama 1: Flujo de entrada al CARA UC

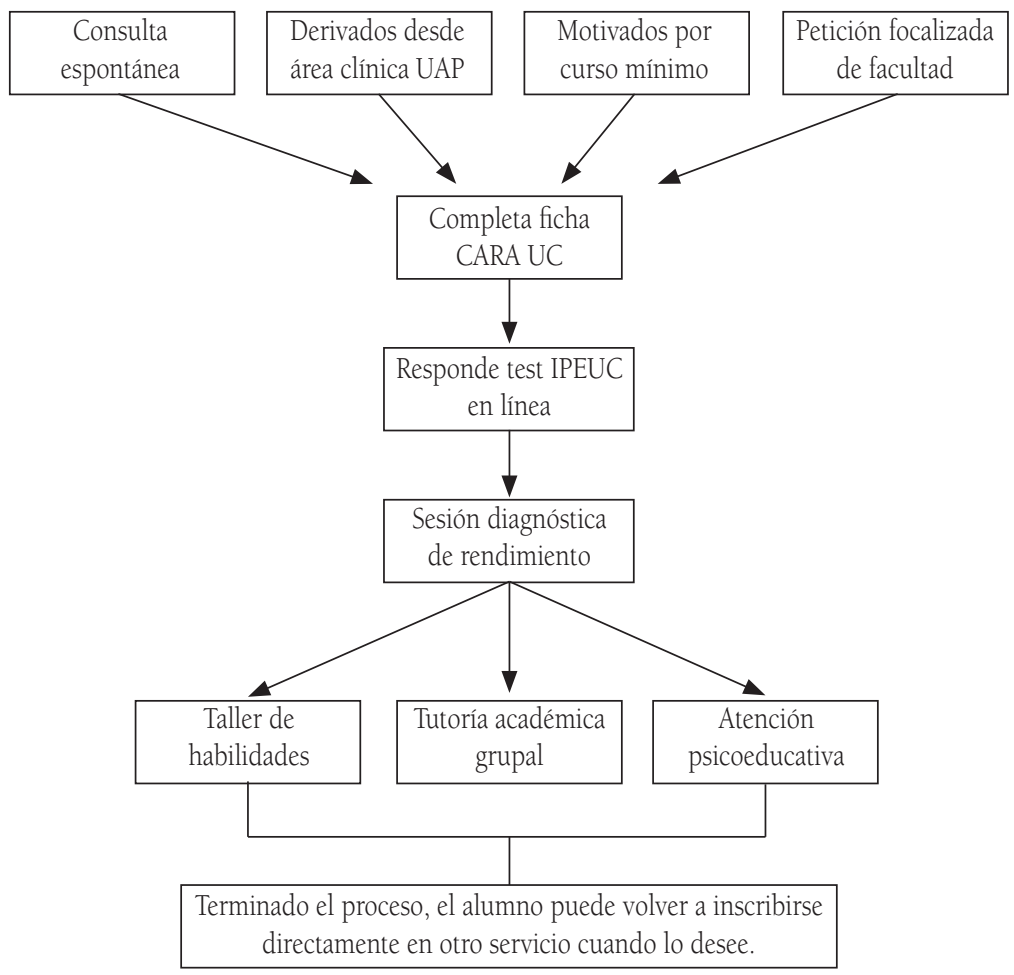

Los estudiantes que acceden al CARA UC deben, en primer lugar, completar una ficha de atención y responder el test IPEUC en línea. Con estos antecedentes, deben asistir a la sesión diagnóstica de rendimiento para conocer sus resultados y las alternativas de desarrollo que les ofrece el Centro. Sólo entonces los alumnos pueden inscribirse y participar en el servicio que deseen. Al finalizar el proceso, se les solicita que lo evalúen. 
El CARA UC cuenta con indicadores generales en relación con su funcionamiento y con datos respecto del grado de satisfacción de los alumnos participantes, los cuales serán revisados para aquellos servicios donde los estudiantes asisten de manera espontánea (en concordancia con la muestra de este estudio).

En primer lugar, los alumnos muestran una total satisfacción con cada uno de los servicios en los que participaron (taller, tutoría o atención psicoeducativa): el 100\% recomendaría el servicio a un amigo.

Por otra parte, el 74\% de los alumnos que asiste a sesión diagnóstica continúa en otro servicio del CARA UC, dando cuenta que el Centro responde en gran medida a sus demandas. Un grupo importante de alumnos acude a la sesión diagnóstica con el único interés de recibir una interpretación de sus resultados en el test de habilidades académicas y otro no se inscribe en un servicio por incompatibilidad horaria. Asimismo, el número de sesiones en este servicio ha aumentado con los años, registrándose un total de 117 sesiones entre 2005 y 2006.

Se han realizado 45 versiones de distintos talleres orientados al desarrollo de habilidades para el aprendizaje y desempeño en la universidad. En éstos, todos los estudiantes manifiestan estar "de acuerdo" o "muy de acuerdo" con los contenidos tratados, el cumplimiento de los objetivos y con los aspectos relativos al facilitador a cargo.

Por otra parte, se ha visto un aumento de los alumnos tutores (actualmente con un promedio de 18) y de las asignaturas para las cuales se ofrece tutoría, incorporando paulatinamente ramos de distintas carreras, principalmente del área científica. Hasta la fecha se ha realizado un total de 1.942 sesiones de asesoría técnica. Respecto de la satisfacción de los alumnos con este servicio, los tutores son evaluados en promedio con nota 6,8 (en escala de 1 a 7) por los participantes, valorando tanto su acogida y calidad pedagógica como el dominio de los contenidos. Además, la gran mayoría (82\%) de 
318 APOYO ACADÉMICO EN LA EDUCACIÓN SUPERIOR - María Inés Susperreguy, Alejandra Flores, Sonia Micin, M. Soledad Zuzulich

los alumnos que asistieron el segundo semestre de 2006 aprobó la asignatura en la cual recibió apoyo técnico (no se tiene información sobre los semestres anteriores). Este dato es aún más relevante si se considera que estos alumnos acuden a este servicio cuando presentan un rendimiento académico muy deficiente en el ramo o se sienten inseguros y en riesgo de reprobar.

Por último, el CARA UC ha ido incorporando, de manera gradual, otros indicadores de evaluación de los distintos servicios ofrecidos. Por ejemplo, se tomó una muestra de los alumnos que habían completado su proceso de atención psicoeducativa y se comparó su perfil de rendimiento previo a la intervención (habilidades personales y recursos para el aprendizaje, evaluados a través del test IPEUC antes de iniciar el proceso) con el resultado obtenido al finalizar el trabajo focalizado. Se encontró una mejora significativa en sus estrategias de estudio y habilidades académicas. Asimismo, los alumnos que participaron de un proceso de exploración vocacional luego del trabajo psicoeducativo aumentaron su claridad vocacional y profesional, su motivación y su grado de satisfacción con el rendimiento obtenido.

\section{Metodología}

La presente investigación corresponde a un estudio de carácter exploratorio, dados los escasos antecedentes empíricos de investigación disponibles en Chile, y da cuenta de la población universitaria que consulta por temas vinculados al rendimiento académico. El diseño es de tipo descriptivo, ya que su propósito es representar las principales características de los alumnos que participan en CARA UC y dar cuenta de sus principales motivaciones y de los servicios de apoyo que solicitan. Asimismo, el diseño de investigación es no experimental, ya que no existe una manipulación intencionada de variables, sino sólo la medición de las características de interés.

En el estudio participaron 1.226 estudiantes, que corresponden a todos los que ingresaron espontáneamente al CARA UC durante 2005 y 2006, en cualquiera de sus servicios. 
Ellos completaron la ficha de atención de manera individual al momento de inscribirse, la que recoge características sociodemográficas de los alumnos (género, edad, comuna de residencia), historia escolar (año de egreso y promedio de notas de enseñanza media, promedio obtenido en las pruebas de selección universitaria, antecedentes de repitencia escolar, historia universitaria previa) y antecedentes académicos en la PUC (medio y año de ingreso, carrera que cursa, beneficios socioeconómicos, promedio ponderado acumulado a la fecha de ingreso al Centro, número de ramos cursados, número de ramos reprobados, causales de eliminación, semestres suspendidos). También se solicita información relativa a las motivaciones para participar en el CARA UC (motivo por el cual consulta, servicios en los cuales ha participado y tipos de atenciones). Por último, la ficha incluye algunas preguntas que evalúan la percepción del alumno respecto de algunas variables afectivas y motivacionales relacionadas con el rendimiento académico universitario, por ejemplo, autoevaluación de su claridad vocacional, motivación por la carrera, rendimiento obtenido y claridad del proyecto profesional, así como también el porcentaje estimado de asistencia a clases y ayudantías.

La información fue codificada e ingresada en una base de datos para su posterior análisis descriptivo, con el fin de conocer cómo es la población que consulta espontáneamente al CARA UC.

\section{Resultados}

El Centro ha recibido un total de 1.226 estudiantes, quienes han consultado de manera espontánea. La distribución según año muestra un aumento en el número de alumnos en 2006 (n=648) respecto de 2005 ( $\mathrm{n}=578)$. Las principales características sociodemográficas, académicas y afectivo-motivacionales de ellos se describen a continuación.

\section{Características sociodemográficas de los alumnos}

Respecto de la variable género, no se aprecian grandes diferencias. El 47,5\% son hombres y el 52,5\% son mujeres, distribución similar 
a la presentada por la totalidad de alumnos que rindieron la Prueba de Selección Universitaria (PSU) e ingresaron a la PUC en 2006 (Valdivieso, Antivilo, Barrios, 2006). No obstante, llama la atención que esta distribución varía según el año considerado en el análisis, de forma que el 2005 asistió un porcentaje levemente superior de mujeres (55,7\%) que de hombres (44,3\%) en comparación con 2006, en el que esta diferencia desaparece, siendo representados hombres y mujeres en una proporción cercana al 50\%.

Al considerar la edad de los alumnos participantes, se aprecia que un porcentaje cercano al 50\% tiene 19 años o menos, aunque también acuden alumnos de cursos superiores (cerca del 40\% del total). Sin embargo, la participación de estos últimos decrece a medida que avanza el proceso académico y tiende a concentrarse en los servicios más focalizados como, por ejemplo, en atención psicoeducativa.

Acuden alumnos provenientes de la mayoría de las comunas que componen la Región Metropolitana (RM). La mayor parte reside en la zona oriente $(38,8 \%)$, seguida por el grupo proveniente de la zona sur $(31,8 \%)$ y, en tercer lugar el de la zona poniente $(12,9 \%)$. Además, un porcentaje cercano al 3\% de alumnos señala proceder de alguna comuna cercana a la RM (por ejemplo, Rancagua, San Antonio); no obstante, puede que este número sea mayor al señalado, ya que muchos alumnos provenientes de regiones más distantes han debido trasladarse de comuna para estudiar en esta universidad (porcentaje cercano al 12\% en 2006, según Valdivieso et al., 2006).

\section{Historia escolar de los alumnos}

La gran mayoría de los participantes (68\%) egresó de enseñanza media entre 2003 y 2005. De éstos, cerca del 50\% egresó en los dos últimos años. Sólo un 10\% finalizó su enseñanza media en 2002 y un porcentaje menor en años anteriores.

Apenas un 2\% $(n=24)$ presenta antecedentes de repitencia 
escolar en su enseñanza básica o media, y un 19\% ha tenido estudios previos en otra universidad o en otra carrera distinta $(n=229)$.

En su conjunto, los alumnos que asisten al CARA UC egresaron de enseñanza media con un promedio de notas de 6,27 (DS=0,35) y obtuvieron un promedio aproximado de 693,4 (DS=50,15) en las pruebas de selección universitaria, resultado similar al puntaje promedio de ingreso a la PUC de todos los alumnos en 2006 (referencia de 694,2 puntos en Valdivieso et al., 2006). Es decir, los alumnos que asisten presentan resultados que denotan excelencia académica en relación con los indicadores de nuestro país.

\section{Antecedentes académicos en la PUC}

El 81,1\% de los alumnos que ingresan al CARA UC lo hace mediante las pruebas de selección universitaria (PSU/PAA). Los demás alumnos lo han hecho por otras medios (por ejemplo, deportista destacado, admisión especial, posgrado y alumno de intercambio).

Si bien acuden alumnos de todos los niveles educativos, la gran mayoría son de primer o segundo año $(65,2 \%)$. Los que cursan segundo año de universidad se presentan en una proporción levemente superior, la cual baja drásticamente cuando se trata de alumnos de tercer año (13,5\%). Si bien también participan alumnos con cuatro, cinco o seis años de universidad, su proporción es muy pequeña (en torno al $4 \%$ en cada caso).

En 2006 ingresaron un total de 2.394 alumnos a la PUC mediante las pruebas de selección universitaria (Valdivieso et al, 2006), de los cuales aproximadamente el $16 \%$ ha asistido al CARA UC ( $\mathrm{n}=382$ ). Además, los alumnos que participan de forma obligatoria en los cursos mínimos de "Hábitos y estrategias de estudio", que se dictan en algunas facultades, fueron 815 en 2006. Por lo tanto, sumando ambas poblaciones se podría afirmar que el CARA UC recibió en alguna de sus modalidades a la mitad de los estudiantes que ingresaron a la UC ese año $(n=1.197)$. 
322 APOYO ACADÉMICO EN LA EDUCACIÓN SUPERIOR - María Inés Susperreguy, Alejandra Flores, Sonia Micin, M. Soledad Zuzulich

$\mathrm{Al}$ analizar la carrera de procedencia, se puede apreciar que la mayoría proviene del área científica y, en segundo lugar, de las ciencias sociales, si bien la participación de alumnos de otras unidades académicas también es relevante. De hecho, participan alumnos de todas las facultades (incluyendo alumnos de posgrado), lo que da cuenta de la difusión que ha tenido el Centro en la universidad.

De acuerdo con los datos obtenidos de las fichas de atención, aproximadamente el $24 \%$ de los alumnos ( $n=293$ ) informa contar con algún beneficio para financiar su arancel o gastos asociados a los estudios, otorgados por la PUC o el Estado, principalmente. La mayoría cuenta con Beca de Alimentación ( $\mathrm{n}=66)$, Beca Bicentenario ( $\mathrm{n}=59$ ), Beca Juan Gómez Millas ( $\mathrm{n}=40$ ) o Beca Padre Hurtado ( $\mathrm{n}=30$ ). Además, de la totalidad de alumnos que participa en el Centro y tiene beneficios, un $8 \%$ cuenta con un segundo beneficio socioeconómico y un $4 \%$ con un tercer beneficio. No se tiene antecedentes respecto del número de alumnos que financia sus estudios con algún crédito bancario o de otro tipo.

Para revisar los resultados académicos obtenidos por los alumnos en la PUC al momento de ingresar al CARA UC, se analizó el Promedio Ponderado Acumulado (PPA), que considera todas las asignaturas cursadas por el estudiante con su respectiva ponderación en el promedio final. Este indicador se puede obtener sólo para aquellos que se encuentren al menos en el segundo semestre de universidad, puesto que los que cursan su primer semestre no tienen aún promedios finales.

Si bien las notas en la educación superior varían entre las carreras y dentro de una misma, en conjunto los alumnos tienen un PPA de 4,59 (DS=0,66) y han cursado un promedio de 14 cursos $(\mathrm{DS}=12,51)$ al momento de su inscripción (la mayoría son alumnos de primeros años). De los ramos inscritos, los estudiantes han reprobado cinco en promedio (DS=5,25). El 19,5\% reprueba sólo un ramo, el $17,3 \%$ dos y un porcentaje cercano al 11\% reprueba tres, 
cuatro y cinco, respectivamente. Los porcentajes de reprobación para mayor cantidad de asignaturas son significativamente inferiores.

Apenas un $6 \%$ de los alumnos que asiste al CARA UC ha suspendido al menos un semestre académico durante su carrera $(\mathrm{n}=77)$. De éstos, el 88,3\% suspendió sólo un semestre académico y un 10,4\% dos. Entre estos últimos, aproximadamente el 60\% recibió la suspensión por motivos de salud y el 22\% por medio de la Dirección de Servicios y Registros Docentes de la universidad.

Sólo un 10,4\% ha estado en riesgo de ser eliminado por motivos académicos $(\mathrm{n}=128)$. La PUC cuenta con un procedimiento para aquellos alumnos que no cumplan con los requisitos académicos mínimos establecidos por cada facultad, ante lo cual ofrece dos instancias de apelación: primero, una comisión de su facultad y, luego, una Comisión de Gracia desde el nivel de la institución.

\section{Motivaciones para participar en el CARA UC}

La mayoría señala directamente que asiste para optimizar su rendimiento académico; desean que éste aumente porque no cumple con sus expectativas, han reprobado ramos o están en riesgo de reprobar, entre otros motivos (ver tabla 1). Además, un grupo importante manifiesta la necesidad de conocer, aprender, perfeccionar y/o potenciar sus hábitos, métodos y estrategias de estudio. También acude un grupo significativo de estudiantes solicitando ayuda específica en ciertos ramos, a través de las tutorías académicas que ofrece el Centro.

Los alumnos mencionan también como necesidad aprender a gestionar y planificar el tiempo, desarrollar y mejorar ciertos procesos cognitivos (en especial concentración y memoria), explorar su vocación y aumentar su motivación académica. Además, señalan su interés por talleres específicos, por el desarrollo de otras habilidades y cierta preocupación por síntomas asociados al rendimiento, fundamentalmente ansiedad y estrés (estos últimos incluidos en "otros motivos de consulta"). 
324 APOYO ACADÉMICO EN LA EDUCACIÓN SUPERIOR - María Inés Susperreguy, Alejandra Flores, Sonia Micin, M. Soledad Zuzulich

Tabla 1. Motivos de consulta de los alumnos que participan en el CARA UC

\begin{tabular}{l|c|c}
\hline Categoría & $\mathrm{N}^{0}$ de menciones & $\%$ \\
\hline Rendimiento & 417 & 28,1 \\
\hline Hábitos y estrategias de estudio & 224 & 15,1 \\
\hline Tutorías en ramos específicos & 168 & 11,3 \\
\hline Concentración y memoria & 135 & 9,1 \\
\hline Manejo y planificación del tiempo & 97 & 6,5 \\
\hline Exploración vocacional & 93 & 6,3 \\
\hline Motivación académica & 66 & 4,5 \\
\hline Otros tema psicológicos ${ }^{2}$ & 58 & 3,9 \\
\hline Orientación y búsqueda de información general & 48 & 3,2 \\
\hline Otros motivos de consulta & 177 & 12,1 \\
\hline Total & 1.483 & 100 \\
\hline
\end{tabular}

Considerando la población total de alumnos que ingresa al Centro, se puede observar que más de la mitad acude a la sesión diagnóstica de rendimiento ( $\mathrm{n}=638$ ), más de un 30\% a tutorías académicas y un $25 \%$ a cursos de "Hábitos y estrategias de estudio". Los demás servicios concentran un porcentaje cercano al 15\%.

Al analizar la participación en los distintos servicios a la luz del año estudiado, en 2006 se aprecia un aumento en los participantes de los cursos de "Hábitos y estrategias de estudio", en los talleres de habilidades académicas y en la atención psicoeducativa.

Del total que ha participado en talleres, un $10 \%(\mathrm{n}=22)$ realizó, a su vez, un segundo taller CARA UC, es decir, continuó profundizando en otro tema de interés. Los talleres más demandados son "Rendimiento académico" (69\%) y "Concentración y memoria" $(18,6 \%)$.

Respecto de las tutorías académicas, si bien la cantidad de estudiantes por año se ha mantenido, en 2006 aumentó el número de veces que un alumno asistió; es decir, en promedio, los alumnos acudieron a más sesiones, aumentando la tasa de retención. La tutoría más solicitada es la de Cálculo I (33,7\%), luego Álgebra

2 Por ejemplo, autoestima, problemas de sueño, adaptación a la universidad. 
(25,2\%), Geometría (19,5\%) y Química General (18,1\%). Además, aproximadamente el $25 \%$ de los que realizan una primera tutoría continúa con una segunda $(\mathrm{n}=102)$.

De manera similar, en 2006 los alumnos que participaron de la atención psicoeducativa asistieron en promedio a cuatro sesiones, con una alta adherencia. Esto puede ser confirmado con los resultados de la evaluación del proceso, donde el 100\% de los alumnos recomendaría este servicio a un amigo ${ }^{3}$.

Al revisar los antecedentes de atención clínica y/o psiquiátrica en la UAP, se encuentra que un $11 \%$ de quienes participan del CARA UC $(n=135)$ ha tenido consulta psiquiátrica y un $25 \%(n=304)$ ha consultado a un psicólogo clínico. Un porcentaje menor ha participado de algún taller preventivo en temas de salud mental $(n=14)$.

\section{Percepción del alumno respecto de variables afectivo-motivacionales vinculadas al rendimiento académico}

La gran mayoría de los que asiste al CARA UC informa estar insatisfecho con su rendimiento actual (74\%), a pesar de encontrarse motivados y sin dudas vocacionales. De hecho, un gran número declara estar satisfecho con la claridad de su proyecto profesional (68\%), con su motivación por la carrera actual (63\%) y con su claridad vocacional (68\%) (ver tabla 2). De manera congruente, un 10\% señala poseer poca claridad respecto de su vocación, un porcentaje cercano al 9\% reporta una baja certeza de su proyecto profesional y motivación con la carrera actual.

3 Los demás servicios muestran resultados similares. 
326 APOYO ACADÉMICO EN LA EDUCACIÓN SUPERIOR - María Inés Susperreguy, Alejandra Flores, Sonia Micin, M. Soledad Zuzulich

Tabla 2. Percepción del alumno respecto de variables vinculadas al rendimiento

\begin{tabular}{|c|c|c|c|c|c|c|c|c|c|}
\hline Grado de satisfacción & Muy & to/a & & & & & Muy & ajo/a & Total \\
\hline & $\mathrm{N}$ & $\%$ & $\mathrm{n}$ & $\%$ & $\mathrm{n}$ & $\%$ & $\mathrm{n}$ & $\%$ & $\mathrm{n}$ \\
\hline Claridad de la vocación & 325 & 32,3 & 359 & 35,7 & 219 & 21,8 & 102 & 10,1 & 1.005 \\
\hline $\begin{array}{l}\text { Motivación con la carrera } \\
\text { actual }\end{array}$ & 243 & 24,2 & 391 & 39,0 & 282 & 28,1 & 87 & 8,7 & 1.003 \\
\hline Rendimiento & 19 & 2,0 & 229 & 24,0 & 558 & 58,5 & 148 & 15,5 & 954 \\
\hline $\begin{array}{l}\text { Claridad de la proyección } \\
\text { profesional }\end{array}$ & 252 & 25,2 & 415 & 41,5 & 247 & 24,7 & 87 & 8,7 & 1.001 \\
\hline
\end{tabular}

\section{Porcentaje de asistencia a clases y ayudantías}

Otra de las variables clave sobre rendimiento académico es el grado en que los alumnos asisten a clases y ayudantías. Al respecto, los alumnos del CARA UC afirman asistir en promedio al 86,7\% de las clases de sus mallas curriculares (DS=15,13). De éstos, el 64\% señala que asiste al menos al $90 \%$ y sólo un $15 \%$ a menos de un $75 \%$ de las clases.

Respecto de la asistencia a las ayudantías, el promedio es de un $82,06 \%(D S=21,72$ ), porcentaje levemente inferior al de asistencia a clases, si bien presenta una heterogeneidad mayor. De éstos, el 59\% de los alumnos asiste a un $90 \%$ o más de las ayudantías y un $24 \%$ a menos de un $75 \%$.

En síntesis, respecto de la población que participa en el CARA $\mathrm{UC}$, los resultados indican que:

- No se aprecian grandes diferencias por género, es decir, asiste una proporción similar de hombres y mujeres.

- Aproximadamente la mitad de los alumnos tiene 19 años de edad o menos, lo cual demuestra que la mayoría de los participantes cursa sus primeros años de universidad, aunque los demás grupos etarios también se encuentran representados.

- Participan alumnos que residen en todas las zonas geográficas de la Región Metropolitana, destacándose los grupos de la zona oriente, sur y poniente, respectivamente.

- Los alumnos no presentan antecedentes académicos de repitencia escolar significativa e ingresan a la universidad con un promedio 
PSU similar a la población total de alumnos que ingresa a la PUC por admisión regular.

- El grupo de alumnos que se acerca al CARA UC y participa de sus servicios constituye un porcentaje significativo de la población universitaria "novata".

- Asisten alumnos de todas las carreras de la universidad, aunque destacan los del área científica y ciencias sociales.

- Casi un cuarto de los participantes en el programa cuenta con algún beneficio económico o beca para financiar sus estudios o los gastos asociados a éstos (sin considerar créditos universitarios ni bancarios).

- Sólo una décima parte de los alumnos ha estado en riesgo real de ser eliminado de la universidad debido a su rendimiento, habiendo superado un proceso de causal de eliminación.

- Los alumnos acuden tanto para pedir apoyo frente a experiencias de bajo rendimiento como para fortalecer y enriquecer sus estrategias de estudio, es decir, acuden en busca de servicios remediales y de prevención en forma paralela.

- Todos los servicios ofrecidos son altamente demandados, destacando el interés de los alumnos por la sesión diagnóstica de rendimiento y las tutorías académicas.

- En relación con las variables afectivo-motivacionales de los participantes, si bien la gran mayoría declara poseer claridad vocacional y profesional, y motivación por su carrera, sólo un cuarto está satisfecho con su rendimiento.

- La gran mayoría de los alumnos declara asistir en un alto porcentaje a clases y ayudantías.

\section{Discusión}

Lo primero que llama la atención es el alto interés que muestran los alumnos de la PUC por el CARA UC. El hecho de que un 16\% de los "novatos" se acerque voluntariamente y participe de alguno de sus servicios, revela que el Centro responde a una necesidad de apoyo académico sentida por los estudiantes, incluso antes de cursar su 
328 APOYO ACADÉMICO EN LA EDUCACIÓN SUPERIOR - María Inés Susperreguy, Alejandra Flores, Sonia Micin, M. Soledad Zuzulich

primer semestre en la universidad (es decir, sin antecedentes de "fracaso académico"). Si se considera el alto porcentaje de asistencia a clases y ayudantías declarado por los alumnos, se podría suponer que, a pesar de que se comportan con responsabilidad ante las demandas académicas, ello no parece suficiente para obtener buenos resultados. Por otro lado, el aumento en la participación de los alumnos en el Centro en 2006 y la diversidad de carreras de procedencia se ha dado de manera espontánea, difundiéndose principalmente a través de los alumnos participantes.

La población del CARA UC no presenta grandes diferencias con el resto de la población universitaria seleccionada por la PSU. Es decir, los alumnos que aquí participan tendrían antecedentes académicos previos al ingreso a la universidad similares a sus compañeros que no participan. Este dato es relevante, ya que, desde sus inicios, el Centro quiso proyectarse hacia la universidad como una alternativa de desarrollo académico para todos los que quisieran mejorar sus estrategias de aprendizaje y no sólo convocar a aquellos con "mal rendimiento". En este sentido, muestra signos de consolidación como una intervención de carácter preventivo, lo cual ha sido valorado por los estudiantes a través de la alta demanda de sus servicios.

Para el Centro, este tipo de prevención apunta a promover el bienestar general y salud mental de los estudiantes, en tanto el rendimiento constituye para ellos un factor particularmente sensible en esta etapa de la vida. Lo anterior, revela la importancia de la intervención en los primeros años (intervención temprana) de modo de fomentar los recursos individuales y prevenir la deserción futura. Los datos indican que el CARA UC se ha perfilado no sólo como un organismo para el apoyo académico, sino también para la adaptación a la vida universitaria, donde además del desarrollo de habilidades académicas, los estudiantes buscan un espacio de integración y orientación general respecto de su experiencia universitaria: acompañamiento en procesos de exploración vocacional, apoyo y derivación ante sintomatología específica causada por la presión académica, entre otros aspectos. 
Los indicadores de rendimiento previo y de selección no garantizan necesariamente el éxito académico ni una adecuada adaptación a la vida universitaria. Por esta razón, las universidades deben explorar y profundizar las necesidades educativas de sus alumnos, y considerar que los indicadores cuantitativos entregan datos generales pero no de los procesos a través de los cuales éstos aprenden. Para conocer a la población y ofrecer adecuadas alternativas de desarrollo, es necesario considerar tanto los procesos de aprendizaje como los resultados académicos. Por otro lado, la gran heterogeneidad de carreras y motivos de consulta que presentan los participantes en el CARA UC releva la importancia de trabajar enfocado en el desarrollo de habilidades, por sobre el trabajo con contenidos específicos. Las materias son responsabilidad de cada facultad y son éstas quienes ofrecen alternativas para su aprendizaje (clases, ayudantías, seminarios, coloquios, por ejemplo). Sin embargo, el desarrollo de habilidades académicas trasciende tanto a las facultades como a los problemas de bajo rendimiento, por lo que intervenciones como la del CARA UC son generalizables a estudiantes de distintas carreras, perfiles de selección y problemas puntuales.

Con relación a los servicios del Centro, se evidencian ciertas diferencias en la distribución de los participantes: las tutorías privilegian la participación de alumnos de carreras científicas y de primer año (debido al énfasis en asignaturas de alta reprobación) y la atención psicoeducativa recibe estudiantes mayores y con problemas más específicos. Por su parte, la población que participa en los talleres es más heterogénea, aunque muestra una tendencia a concentrar alumnos de los primeros tres años de universidad. Ante este resultado, es necesario recalcar la necesidad de tener servicios variados para cubrir sus demandas e ir adaptando las ofertas según las oportunidades. La experiencia de contar con un equipo de psicólogos educacionales a cargo de los servicios ha sido positiva, en tanto poseen una visión amplia de la influencia de variables psicológicas y educativas, posiblemente relacionadas con el rendimiento, trabajando desde una perspectiva basada en los recursos del estudiante. Es importante contextualizar que el CARA UC surge inserto en una 
330 APOYO ACADÉMICO EN LA EDUCACIÓN SUPERIOR - María Inés Susperreguy, Alejandra Flores, Sonia Micin, M. Soledad Zuzulich

unidad de apoyo psicológico, cuyo foco es la promoción y prevención vinculada al rendimiento como factor protector de la salud mental. Lo anterior permite una relación oportuna y estrecha con el área clínica para abordar los problemas desde una perspectiva integral. Esta alianza constituye una instancia de trabajo multidisciplinario entre distintas áreas de la psicología, la que, a su vez, se enriquece incorporando a otros profesionales vinculados al rendimiento en beneficio de los alumnos. Por otro lado, la alianza de trabajo y el contacto permanente con las autoridades de las distintas facultades de la universidad ha sido crucial para convocar a los alumnos y ofrecer intervenciones oportunas. Este tipo de alianzas ha dado origen a servicios específicos en beneficio de estudiantes con distintos conflictos y necesidades de apoyo.

Entre los desafíos y proyecciones del Centro se puede mencionar, en primer lugar, la necesidad de perfeccionar y diseñar nuevos mecanismos de evaluación de resultados de las intervenciones. Hasta ahora los datos indican que el interés de los alumnos por optimizar su proceso de estudio es alto, que los servicios ofrecidos son atingentes y que el nivel de satisfacción de los participantes con dichos servicios es altísimo. Sin embargo, es preciso avanzar en la medición del impacto de los diferentes servicios CARA UC, tanto en el plano cuantitativo (por ejemplo, promedio de notas, aprobación de ramos) como cualitativo (habilidades, procesos y recursos personales). Del mismo modo, es urgente incorporar más y mejores instrumentos para evaluar diferenciadamente la efectividad de las intervenciones y la satisfacción de los alumnos una vez finalizada su participación. En ambos casos se debe buscar integrar tanto indicadores duros como blandos.

Por otro lado, en el proceso de seguimiento aparece la enorme complejidad de evaluar el impacto de las intervenciones en el rendimiento expresado en notas, ya que este indicador está influenciado por diversas variables externas a los servicios del Centro $e$, incluso, ajenas a los propios alumnos en algunos casos (por ejemplo, calidad docente, currículo, sistemas de evaluación). El desafío 
de evaluar las intervenciones debe trascender este dato y debería estar orientado a construir un indicador pertinente e integrador de la realidad del alumno y las características de la intervención. A modo de seguimiento, el CARA UC ha modificado y actualizado sus instrumentos de evaluación, ha incorporado nuevos indicadores de proceso y de resultado, y ha iniciado diversos estudios para validar las estrategias implementadas y/o dar luces sobre nuevas necesidades. Asimismo, pretende realizar cada vez más actividades de extensión con la intención de compartir y poner al servicio de otras instituciones su experiencia en este campo, así como, también, conocer y aprender de la experiencia de otras entidades con intervenciones similares. En Chile, las universidades parecen tener una función irremplazable en el abordaje de las necesidades académicas de sus estudiantes y una oportunidad única para mejorar la calidad y equidad de la educación. El desarrollo e implementación de programas preventivo/remediales y de carácter psicoeducativo son una alternativa viable para contribuir a compensar las diferencias de entrada en sus alumnos y ofrecerles herramientas concretas que los ayuden a rendir y superar con éxito los desafíos que enfrentan.

Con el fin de promover e implementar políticas educacionales nacionales que comprometan la incorporación de la enseñanza y/o entrenamiento de habilidades académicas en el ámbito de la educación superior, es necesario potenciar espacios de colaboración y trabajo conjunto entre las instituciones que, si bien son particulares y reciben poblaciones diferentes, comparten el objetivo de educar y formar al capital humano en Chile con mayor equidad.

\section{Referencias bibliográficas}

Alvarado, Hugo; Sánchez, Iván y Uribe, Marco. (2000) Correspondencia entre estrategias de aprendizaje y rendimiento académico en estudiantes universitarios. Boletín de Investigación Educacional, 15, pp. 70-88.

Andrews, Bernice y Wilding, John. (2004) The relation of depression and anxiety to life-stress and achievement in students. British Journal of Psychology, 95(4), pp. 509-521. 
332 APOYO ACADÉMICO EN LA EDUCACIÓN SUPERIOR - María Inés Susperreguy, Alejandra Flores, Sonia Micin, M. Soledad Zuzulich

Badenier, Carolina. (2003) Confiabilidad y validez del Learning and Study Strategies Inventory (LASSI) en una muestra de estudiantes de la Región Metropolitana. Psykhe, 12(2), pp.193-206.

Bilbao, Gilda. (2004) Estrategias de Aprendizaje. Aplicación de un Programa de Enseñanza, basado en la adquisición de estrategias de aprendizaje, en un grupo de estudiantes universitarios. Tesis para optar al grado de Magíster en Educación Especial, Facultad de Educación, Pontificia Universidad Católica de Chile, Santiago, Chile.

Brunner, José Joaquín y Elacqua, Gregory. (2003) Informe Capital Humano en Chile. Santiago, Chile: Universidad Adolfo Ibáñez, Escuela de Gobierno.

Consejo Superior de Educación, Chile. INDICES 2007. Disponible en http://indices.cse.cl/indices/index.asp Último acceso el 25 de mayo de 2007.

Díaz, Francisco; Prado, Mercedes y López, Santiago. (2003) Relación entre rendimiento académico, síntomas depresivos, edad y género en población de adolescentes. Psicothema, 12(4), pp. 525-532.

Estévez, Etty. (2002) Enseñar a pensar: ¿Nuevo enfoque de la educación? Monterrey: ITESM.

García, Fernando y Ramírez, Patricio. (2005) Caracterización de las estrategias de aprendizaje en estudiantes en formación de Pedagogía en Educación General Básica. Boletín de Investigación Educacional, 20(2), pp. 189210.

Gómez, Bernardo; Osorio, Lidia; Domínguez, Paula; Silva, María Gabriela; Navea, Dagoberto; Monárdez, Marta y Silva, Angélica. (2002) Programa de desarrollo académico para alumnos de primer año. Calidad en la Educación, 17(2), pp. 251 - 267.

Kolb, David. (1976) The Learning Style Inventory: Technical Manual. Boston, Ma.: McBer.

Manelic, Hugo y Ortega Soto, Héctor. (1995) La depresión en estudiantes universitarios de la Escuela Nacional de Estudios Profesionales Plantel Aragón. Salud Mental, 18(2), pp.31-34.

Polo, Antonia; Hernández, José Manuel y Pozo, Carmen. (1996) Evaluación del estrés académico en estudiantes universitarios. Ansiedad y Estrés, 2(2-3), pp. 159-172.

Sanabria, Hernán. (2002) Deserción en estudiantes de enfermería en cuatro 
universidades del Perú [Versión electrónica]. Anales de la Facultad de Medicina, Universidad Nacional Mayor de San Marcos, 63(4), pp.301311.

Rosas, Ricardo, Manzi, Jorge y Moore, Catalina. (1987) Construcción y estandarización del inventario de procesos de estudio UC (IPEUC). Santiago de Chile: Escuela de Psicología, Universidad Católica de Chile.

Rosso, Pedro Pablo. (2004) Tareas del plan de desarrollo 2005-2010. Tareas $\mathrm{n}^{\circ}$ 1.2.1 y 1.2.7. Pontificia Universidad Católica de Chile.

Valdivieso, Pablo; Antivilo, Andrés y Barrios, Juan. (2006) Caracterización sociodemográfica de estudiantes que rinden la PSU, postulan y se matriculan en Universidades del Consejo de Rectores. Calidad de la Educación, (24), pp. 319-361.

Villarroel, Verónica. (2001) Relación entre autoconcepto y rendimiento académico. Psykhe, 10(1), pp. 3-18.

Recibido: 13 de abril de 2007 Aceptado: 14 de mayo de 2007 